\section{Heißt hohe Dosis unbedingt auch hohe Wirksamkeit?}

\author{
Thema des Sonderhefts 1 von Allergo Journal 2005 (14: S1-28) war \\ „Evidenz für die Wirksamkeit der hoch dosierten sublingualen \\ Immuntherapie“. Einzelne Beiträge dieses Heftes enthielten Aus- \\ sagen, die der Autor des folgenden Leserbriefs nicht unwidersprochen \\ lassen will.
}

\begin{abstract}
$\mathrm{m}$ Editorial [1] des Sonderheftes wird die Hypothese aufgestellt, dass die Tagesdosis an Allergen der wesentliche $\mathrm{Pa}$ rameter zur Wirksamkeitsbeurteilung der sublingualen Immuntherapie (SLIT) sei. Es komme auf eine hohe Dosis bei der SLIT an, die um einen Faktor 50-100 über derjenigen der subkutanen Immuntherapie (SCIT) liegen sollte. Wie wenig hilfreich diese Aussage ist, wird deutlich, wenn man sich vor Augen führt, dass die SCIT-Lösungen verschiedener Hersteller nicht vergleichbar sind. Die scheinbar erforderliche „hohe Dosierung“ eines Produkts zur sublingualen Immuntherapie kann ganz einfach auf einer niedrigen Konzentration des Allergens im Injektionspräparat der gleichen Firma beruhen.

Auch ein Vergleich von SLIT-Produkten, wie er in der Studie „Vergleich der biologischen Potenz verschiedener Allergenextrakte" [2] vorgenommen wurde, kann irreführend sein. Die Schluss-
\end{abstract}

folgerung aus der Studie lautet: „Diese Ergebnisse lassen zwar keinen direkten Rückschluss auf das Ausmaß der Wirksamkeit verschiedener Präparate zu, aber erlauben eine eindeutige Einordnung der Dosierung. Dies könnte für den klinisch tätigen Allergologen bei der therapeutischen Entscheidung hilfreich sein [... $]^{\text {". }}$ Diese Folgerung geht fehl, wie an Hand einer Cochrane-Metaanalyse (siehe unten) zu belegen ist. Die hier publizierte Studie sollte keine Richtschnur für den Kliniker darstellen, vor einfachen Rückschlüssen kann nur gewarnt werden. Stattdessen sollte der Arzt die Literatur zur Wirksamkeit der verschiedenen Produkte kritisch analysieren, um die Erfolgsaussichten abzuschätzen.

In einem Artikel von Malling [3] sind die beiden größten Hersteller von Produkten zur SLIT, Stallergenes und ALKAbelló, mit klinischen Studien vertreten. Nach Malling scheint die tägliche Dosie-

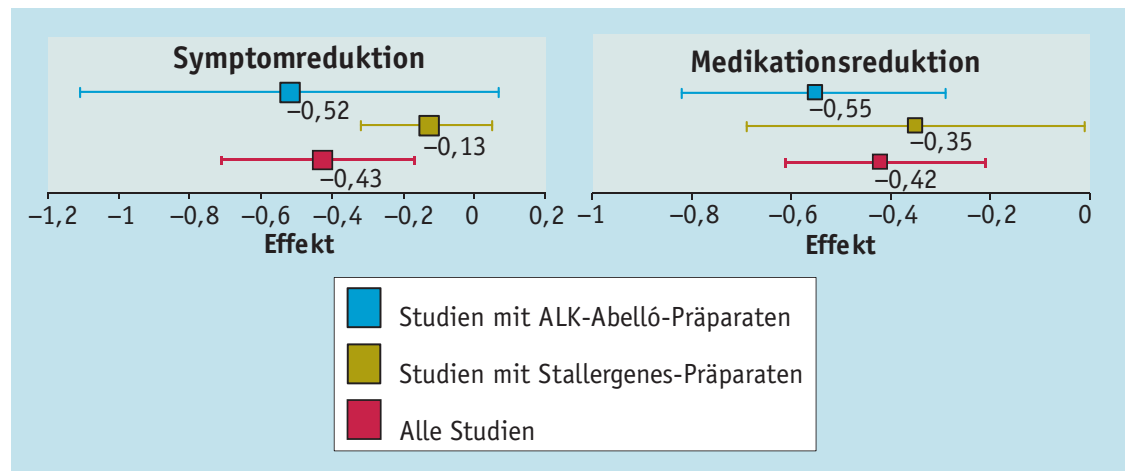

Abbildung. Untergruppenanalyse aus der Cochrane-Metaanalyse [4] für die beiden meist verkauften SLIT-Präparate. Ein Effekt von $-0,2,-0,5$ oder $-0,8$ gibt einen schwachen, moderaten oder starken Effekt an. Der Beitrag der mit StallergenesProdukten durchgeführten Studien („high dose“-Produkte) zum Gesamtergebnis ist deutlich kleiner als der Beitrag der mit ALK-Abelló-Produkten durchgeführten Studien. Zusammengenommen machen die ALK-Abelló- und Stallergènes-Studien mit Bezug auf Symptom- bzw. Medikationscores $80 \%$ bzw. $87 \%$ der erfassten Patienten in der Metaanalyse [4] aus. rung an sich kein Prädiktor der Wirksamkeit zu sein, nur eine von sechs Studien mit dem angeblichen „high dose“-Produkt wurde als wirksam dokumentiert. Weiterhin ist der Beitrag der verschiedenen Produkte zur durchschnittlichen Wirksamkeit in der Cochrane-Metaanalyse von Wilson et al. [4] sehr interessant. Hier zeigt die Analyse der Studien mit Stallergenes-Präparaten eine durchschnittliche Reduktion der Punktescores für Symptome und Medikation von $-0,13$ (95\%-Konfidenzintervall [KI]: $[-0,32 ; 0,5], \mathrm{n}=7)$ und $-0,35$ (95\%-KI: [-0,69;-0,01], $\mathrm{n}=7$ ), während die mit den ALK-Abelló-Produkten durchgeführten Studien eine durchschnittliche Wirksamkeit von -0,52 (95\%-KI: $[-1,11 ; 0,07], \mathrm{n}=8)$ und $-0,55(95 \%-\mathrm{KI}$ : $[-0,81 ;-0,28], \mathrm{n}=7)$ aufweisen (Abb.). Diese Daten belegen, dass offenbar die tägliche Dosierung der SLIT-Produkte an sich - es müsste ja wohl auch eher die kumulative Dosis sein - keinen Hinweis auf die Wirksamkeit gibt. Wie die Metaanalyse eindrücklich bestätigt, muss die klinische Wirksamkeit für jedes Produkt anhand von Daten einzeln nachgewiesen werden - Unterschiede gibt es eindeutig zwischen den Produkten. Man kann darüber nachdenken, wieso die beiden meist verkauften SLIT-Produkte so unterschiedliche Wirksamkeit zeigen. Abschließend ist festzuhalten, dass Allergenextrakte, die von verschiedenen Herstellern produziert werden, unabhängig von theoretischen Überlegungen in direkten Vergleichsstudien geprüft werden müssen, wenn man tatsächlich eine Aussage zur Überlegenheit eines Produktes treffen will. Alles andere ist unwissenschaftlich und irreführend.

\section{Literatur}

1. Merk HF. Alles eine Frage der Dosis? Allergo J 2005; 14: S3-4

2. Mösges R, Schlierenkämper U, Wacker S. Vergleich der biologischen Potenz verschiedener Allegenextrakte. Allergo J 2005; 14: S22-6

3. Malling $\mathrm{HJ}$. Is sublingual immunotherapy clinically effective? Curr Opin Allergy Clin Immunol 2002; 2: 523-31

4. Wilson DR, Torres LI, Durham SR. Sublingual immunotherapy for allergic rhinitis. Cochrane Database Syst Rev 2003: CDo02893

\section{Prof. Dr. Claus Bachert}

Kliniek voor Neus- Keel- en Oorheelkunde UZ Gent

De Pintelaan 185

9000 Gent, Belgien 